\title{
The Cauchy problem for the modified Novikov equation
}

\author{
Xueping $\mathrm{Hou}^{1 *}$ and Yan Zheng ${ }^{2}$
}

\section{${ }^{*}$ Correspondence:}

houxueping123@163.com

${ }^{1}$ College of Mathematics and

Information Science, Henan Normal

University, Xinxiang, Henan 453007,

P.R. China

Full list of author information is

available at the end of the article

\begin{abstract}
In this paper, we are concerned with the Cauchy problem for the modified Novikov equation. By using the transport equation theory and Littlewood-Paley decomposition as well as nonhomogeneous Besov spaces, we prove that the Cauchy problem for the modified Novikov equation is locally well posed in the Besov space $B_{p, r}^{s}$ with $1 \leq p, r \leq+\infty$ and $s>\max \left\{1+\frac{1}{p}, \frac{3}{2}\right\}$ and show that the Cauchy problem for the modified Novikov equation is locally well posed in the Besov space $B_{2,1}^{3 / 2}$ with the aid of Osgood lemma.

MSC: $35 \mathrm{G} 25 ; 35 \mathrm{~L} 05 ; 35 \mathrm{R} 25$
\end{abstract}

Keywords: Cauchy problem; modified Novikov equation

\section{Introduction}

Recently, Zhao and Zhou [1] considered the exact traveling wave solution to the following modified Novikov equation:

$$
u_{t}-u_{t x x}+4 u^{4} u_{x}=3 u u_{x} u_{x x}+u^{2} u_{x x x}
$$

We recall that the Novikov equation

$$
u_{t}-u_{t x x}=u^{2} u_{x x x}+3 u u_{x} u_{x x}-4 u^{2} u_{x}
$$

was discovered by Vladimir Novikov [2] and it possesses the bi-Hamiltonian structure, infinite conservation laws. The well-posedness and blow-up of the Cauchy problem for the Novikov equation in Sobolev spaces and Besov spaces have been investigated by some authors [3-7]. The weak solution of the Cauchy problem for the Novikov equation has been investigated by some authors [4, 5, 8]. Recently, Li and Yan [9] considered the Cauchy problem for the $\mathrm{KdV}$ equation with higher dispersion.

We define $P_{1}(D)=\partial_{x}\left(1-\partial_{x}^{2}\right)^{-1}$ and $P_{2}(D)=\left(1-\partial_{x}^{2}\right)^{-1}$. By using the fact that $G(x)=\frac{1}{2} e^{-|x|}$ and $G(x) * f=\left(1-\partial_{x}^{2}\right)^{-1} f$ for all $f \in L^{2}(\mathbf{R})$ and $G * y=u$, we can rewrite (1.1) as follows:

$$
u_{t}+u^{2} u_{x}+P_{1}(D)\left[\frac{4}{5} u^{5}-\frac{1}{3} u^{3}+\frac{3}{2} u u_{x}^{2}\right]+P_{2}(D)\left[\frac{1}{2} u_{x}^{3}\right]=0, \quad t>0 .
$$

O 2014 Hou and Zheng; licensee Springer. This is an Open Access article distributed under the terms of the Creative Commons Attribution License (http://creativecommons.org/licenses/by/4.0), which permits unrestricted use, distribution, and reproduction in any medium, provided the original work is properly credited. 
Now we consider the following problem:

$$
\begin{aligned}
& u_{t}+u^{2} u_{x}+P_{1}(D)\left[\frac{4}{5} u^{5}-\frac{1}{3} u^{3}+\frac{3}{2} u u_{x}^{2}\right]+P_{2}(D)\left[\frac{1}{2} u_{x}^{3}\right]=0, \quad t>0, \\
& u(x, 0)=u_{0}(x), \quad x \in \mathbf{R} .
\end{aligned}
$$

To the best of our knowledge, the well-posedness and blow-up of the Cauchy problem for (1.3) and (1.4) in Besov spaces are open up to now. More precisely, in this paper, motivated by $[10,11]$, using Littlewood-Paley decomposition and nonhomogeneous Besov spaces, we prove that the Cauchy problem for (1.4) is locally well posed in the Besov space $B_{p, r}^{s}$ with $s>\max \left\{1+\frac{1}{p}, \frac{3}{2}\right\}$ and we give a blow-up criterion.

To introduce the main results, we define

$$
\begin{aligned}
& E_{p, r}^{s}(T)=C\left([0, T] ; B_{p, r}^{s}\right) \cap C^{1}\left([0, T] ; B_{p, r}^{s-1}\right) \quad \text { if } r<\infty, \\
& E_{p, \infty}^{s}(T)=L^{\infty}\left(0, T ; B_{p, \infty}^{s}\right) \cap \operatorname{Lip}\left([0, T] ; B_{p, r}^{s-1}\right)
\end{aligned}
$$

The main results of this paper are as follows.

Theorem 1.1 Let $1 \leq p, r \leq \infty$ and $s>\max \left(\frac{3}{2}, 1+\frac{1}{p}\right)$ and $u_{0} \in B_{p, r}^{s}$. Then there exists a time $T>0$ such that problem (1.3) and (1.4) has a unique solution $u$ in $E_{p, r}^{s}(T)$. The map $u_{0} \rightarrow u$ is continuous from a neighborhood of $u_{0}$ in $B_{p, r}^{s}$ into $C\left([0, T] ; B_{p, r}^{s^{\prime}}\right) \cap C^{1}\left([0, T] ; B_{p, r}^{s^{\prime}-1}\right)$ for every $s^{\prime}<s$. When $r<\infty$, the solution to problem (1.3) and (1.4) is continuous in $E_{p, r}^{s}(T)$.

Theorem 1.2 When $u_{0} \in B_{2,1}^{3 / 2}$, (1.3) and (1.4) is locally well posed in the sense of Hadamard.

The remainder of this paper is organized as follows. In Section 2, we give some preliminaries. In Section 3, we establish local well-posedness of the Cauchy problem for the generalized Camassa-Holm equation in Besov spaces. In Section 4, we prove Theorem 1.2.

\section{Preliminaries}

In this section, the nonhomogeneous Besov spaces and the theory of transport equation which can be seen in [10-13] are presented.

Lemma 2.1 (Littlewood-Paley decomposition) There exists a couple of smooth radial functions $(\chi, \phi)$ valued in $[0,1]$ such that $\chi$ is supported in the ball $B=\left\{\xi \in \mathbf{R}^{n},|\xi| \leq \frac{4}{3}\right\}$ and $\phi$ is supported in the ring $C=\left\{\xi \in \mathbf{R}^{n}, \frac{3}{4} \leq|\xi| \leq \frac{8}{3}\right\}$. Moreover,

$$
\forall \xi \in \mathbf{R}^{n}, \quad \chi(\xi)+\sum_{q \in \mathbf{N}} \phi\left(2^{-q} \xi\right)=1
$$

and

$$
\begin{aligned}
& \operatorname{Supp} \phi\left(2^{-q} \cdot\right) \cap \operatorname{Supp} \phi\left(2^{-q^{\prime}} \cdot\right)=\emptyset \quad \text { if }\left|q-q^{\prime}\right| \geq 2, \\
& \operatorname{Supp} \chi(\cdot) \cap \operatorname{Supp} \phi\left(2^{-q} \cdot\right)=\emptyset \quad \text { if }|q| \geq 1 .
\end{aligned}
$$


Then, for $u \in \mathcal{S}^{\prime}(\mathbf{R})$, the nonhomogeneous dyadic blocks are defined as follows:

$$
\begin{aligned}
& \Delta_{q} u=0 \quad \text { if } q \leq-2, \\
& \Delta_{-1} u=\chi(D) u=\mathcal{F}_{x}^{-1} \chi \mathcal{F}_{x} u, \\
& \Delta_{q} u=\phi\left(2^{-q} D\right)=\mathcal{F}_{x}^{-1} \phi\left(2^{-q} \xi\right) \mathcal{F}_{x} u \quad \text { if } q \geq 0 .
\end{aligned}
$$

Thus $u=\sum_{q \in \mathbf{Z}} \Delta_{q} u$ in $\wp^{\prime}(\mathbf{R})$.

Remark The low frequency cut-off $S_{q}$ is defined by

$$
S_{q} u=\sum_{p=-1}^{q-1} \Delta u=\chi\left(2^{-q} D\right) u=\mathcal{F}_{x}^{-1} \chi\left(2^{-q} \xi\right) \mathcal{F}_{x} u, \quad \forall q \in N .
$$

It is easily checked that

$$
\begin{aligned}
& \Delta_{p} \Delta_{q} u \equiv 0 \quad \text { if }|p-q| \geq 2, \\
& \Delta_{q}\left(S_{p-1} u \Delta_{p} v\right) \equiv 0 \quad \text { if }|p-q| \geq 5, \forall u, v \in \mathcal{S}^{\prime}(\mathbf{R})
\end{aligned}
$$

as well as

$$
\left\|\Delta_{q} u\right\|_{L^{p}} \leq\|u\|_{L^{p}}, \quad\left\|S_{q} u\right\|_{L^{p}} \leq C\|u\|_{L^{p}}, \quad \forall 1 \leq p \leq+\infty
$$

with the aid of Young's inequality, where $C$ is a positive constant independent of $q$.

Definition (Besov spaces) Let $s \in \mathbf{R}, 1 \leq p \leq+\infty$. The nonhomogeneous Besov space $B_{p, r}^{s}\left(\mathbf{R}^{n}\right)$ is defined by

$$
B_{p, r}^{s}\left(\mathbf{R}^{n}\right)=\left\{f \in \mathcal{S}^{\prime}(\mathbf{R}):\|f\|_{B_{p, r}^{s}}=\left\|2^{q s} \Delta_{q} f\right\|_{l^{r}\left(L^{p}\right)}=\left\|\left(2^{q s}\left\|\Delta_{q} f\right\|_{L^{p}}\right)_{q \geq-1}\right\|_{l^{r}}<\infty\right\} .
$$

In particular, $B_{p, r}^{\infty}=\bigcap_{s \in \mathbf{R}} B_{p, r}^{s}$. Let $T>0, s \in \mathbf{R}$ and $1 \leq p \leq \infty$. Define $E_{p, r}^{s}=\bigcap_{T>0} E_{p, r}^{s}(T)$.

Lemma 2.2 Let $s \in \mathbf{R}, 1 \leq p, r, p_{j}, r_{j} \leq \infty, j=1,2$, then:

(1) Topological properties: $B_{p, r}^{s}$ is a Banach space which is continuously embedded in $\varsigma^{\prime}(\mathbf{R})$

(2) Density: $C_{c}^{\infty}$ is dense in $B_{p, r}^{s} \Leftrightarrow 1 \leq p, r<\infty$.

(3) Embedding: $B_{p_{1}, r_{1}}^{s} \hookrightarrow B_{p_{2}, r_{2}}^{\left.s-\frac{1}{p_{1}}-\frac{1}{p_{2}}\right)}$ if $p_{1} \leq p_{2}$ and $r_{1} \leq r_{2}$.

$$
B_{p, r_{2}}^{s_{2}} \hookrightarrow B_{p, r_{1}}^{s_{1}} \text { locally compact if } s_{1}<s_{2} .
$$

(4) Algebraic properties: $\forall s>0, B_{p, r}^{s} \cap L^{\infty}$ is a Banach algebra. $B_{p, r}^{s}$ is a Banach algebra $\Leftrightarrow B_{p, r}^{s} \hookrightarrow L^{\infty} \Leftrightarrow s>\frac{1}{p}$ or $\left(s \geq \frac{1}{p}\right.$ and $\left.r=1\right)$. In particular, $B_{2,1}^{1 / 2}$ is continuously embedded in $B_{2, \infty}^{1 / 2} \cap L^{\infty}$ and $B_{2, \infty}^{1 / 2} \cap L^{\infty}$ is a Banach algebra.

(5) 1-D Moser-type estimates:

(i) For $s>0$,

$$
\|f g\|_{B_{p, r}^{s}} \leq C\left(\|f\|_{B_{p, r}^{s}}\|g\|_{L^{\infty}}+\|f\|_{L^{\infty}}\|g\|_{B_{p, r}^{s}}\right) .
$$


(ii) $\forall s_{1} \leq \frac{1}{p}<s_{2}\left(s_{2} \geq \frac{1}{p}\right.$ if $\left.r=1\right)$ and $s_{1}+s_{2}>0$, we have

$$
\|f g\|_{B_{p, r}^{s_{1}}} \leq C\|f\|_{B_{p, r}^{s_{1}}}\|g\|_{B_{p, r}^{s_{2}}}
$$

(6) Complex interpolation:

$$
\|f\|_{B_{p, r}^{\theta s_{1}+(1-\theta) s_{2}}} \leq\|f\|_{B_{p, r}^{s_{1}}}^{\theta}\|g\|_{B_{p, r}^{s_{2}}}^{1-\theta}, \quad \forall f \in B_{p, r}^{s_{1}} \cap B_{p, r}^{s_{2}}, \forall \theta \in[0,1]
$$

(7) Real interpolation: $\forall \theta \in(0,1), s_{1}>s_{2}, s=\theta s_{1}+(1-\theta) s_{2}$, there exists a constant $C$ such that

$$
\|u\|_{B_{p, 1}^{s}} \leq \frac{C(\theta)}{s_{1}-s_{2}}\|u\|_{B_{p, \infty}^{s_{1}}}^{\theta}\|u\|_{B_{p, \infty}^{s_{2}}}^{1-\theta}, \quad \forall u \in B_{p, \infty}^{s_{1}} .
$$

In particular, for any $0<\theta<1$, we have that

$$
\|u\|_{B_{2,1}^{1 / 2}} \leq\|u\|_{B_{2,1}^{3}-\theta} \leq C(\theta)\|u\|_{B_{2, \infty}^{1 / 2}}^{\theta}\|u\|_{B_{2, \infty}^{3 / 2}}^{1-\theta}
$$

(8) Fatou lemma: if $\left(u_{n}\right)_{n \in \mathbf{N}}$ is bounded in $B_{p, r}^{s}$ and $u_{n} \longrightarrow u$ in $\boldsymbol{s}^{\prime}(\mathbf{R})$, then $u \in B_{p, r}^{s}$ and

$$
\|u\|_{B_{p, r}^{s}} \leq \liminf _{n \rightarrow \infty}\left\|u_{n}\right\|_{B_{p, r}^{s}}
$$

(9) Let $m \in \mathbf{R}$ and $f$ be an $S^{m}$-multiplier (i.e., $f: \mathbf{R}^{n} \rightarrow \mathbf{R}$ is smooth and satisfies that $\forall \alpha \in N^{n}$, э a constant $C_{\alpha}$, s.t. $\left|\partial_{\alpha} f(\xi)\right| \leq C_{\alpha}(1+|\xi|)^{m-|\alpha|}$ for all $\left.\xi \in \mathbf{R}^{n}\right)$. Then the operator $f(D)$ is continuous from $B_{p, r}^{s}$ to $B_{p, r}^{s-m}$. Notice that $P_{1}(D)$ is continuous from $B_{p, r}^{s}$ to $B_{p, r}^{s-1}$ and $P_{2}(D)$ is continuous from $B_{p, r}^{s}$ to $B_{p, r}^{s-2}$.

(10) The usual product is continuous from $B_{2,1}^{-1 / 2} \times\left(B_{2, \infty}^{1 / 2} \cap L^{\infty}\right)$ to $B_{2, \infty}^{-1 / 2}$.

(11) There exists a constant $C>0$ such that the following interpolation inequality holds:

$$
\|f\|_{B_{2,1}^{1 / 2}} \leq C\|f\|_{B_{2, \infty}^{1 / 2}} \ln \left(e+\frac{\|f\|_{B_{2, \infty}^{3 / 2}}}{\|f\|_{B_{2, \infty}^{1 / 2}}}\right) .
$$

Lemma 2.3 (A priori estimates in Besov spaces) Let $1 \leq p, r \leq \infty$ and $s>-\min \left(\frac{1}{p}, 1-\frac{1}{p}\right)$. Assume that $f_{0} \in B_{p, r}^{s}, F \in L^{1}\left(0, T ; B_{p, r}^{s}\right)$ and $\partial_{x} v$ belongs to $L^{1}\left(0, T ; B_{p, r}^{s-1}\right)$ if $s>1+\frac{1}{p}$ or to $L^{1}\left(0, T ; B_{p, r}^{1 / p} \cap L^{\infty}\right)$ otherwise. If $\in L^{\infty}\left(0, T ; B_{p, r}^{s}\right) \cap C\left([0, T] ; f^{\prime}(\mathbf{R})\right)$ solves the following $1-D$ linear transport equation:

$$
\begin{aligned}
& f_{t}+v f_{x}=F, \\
& f(x, 0)=f_{0},
\end{aligned}
$$

then there exists a constant $C$ depending only on $s, p, r$ such that the following statements hold:

(1) If $r=1$ or $s \neq 1+\frac{1}{p}$, then

$$
\|f\|_{B_{p, r}^{s}} \leq\left\|f_{0}\right\|_{B_{p, r}^{s}}+\int_{0}^{t}\|F(\tau)\|_{B_{p, r}^{s}} d \tau+C \int_{0}^{t} V^{\prime}(\tau)\|f(\tau)\|_{B_{p, r}^{s}} d \tau
$$


or hence,

$$
\|f\|_{B_{p, r}^{s}} \leq e^{C V(t)}\left(\left\|f_{0}\right\|_{B_{p, r}^{s}}+\int_{0}^{t} e^{-C V(\tau)}\|F(\tau)\|_{B_{p, r}^{s}} d \tau\right)
$$

with $V(t)=\int_{0}^{t}\left\|v_{x}(\tau)\right\|_{B_{p, r}^{1 / p} L^{\infty}} d \tau$ if $s<1+\frac{1}{p}$ and $V(t)=\int_{0}^{t}\left\|v_{x}(\tau)\right\|_{B_{p, r}^{s-1}} d \tau$ else.

(2) If $s \leq 1+\frac{1}{p}, f_{0}^{\prime} \in L^{\infty}$ and $f_{x} \in L^{\infty}((0, T) \times \mathbf{R})$ and $F_{x} \in L^{1}\left(0, T ; L^{\infty}\right)$, then

$$
\begin{aligned}
& \|f(t)\|_{B_{p, r}^{s}}+\left\|f_{x}(t)\right\|_{L^{\infty}} \\
& \quad \leq e^{C V(t)}\left(\left\|f_{0}\right\|_{B_{p, r}^{s}}+\left\|f_{0 x}\right\|_{L^{\infty}}+\int_{0}^{t} e^{-C V(\tau)}\left[\|F(\tau)\|_{B_{p, r}^{s}}+\left\|F_{x}(\tau)\right\|_{L^{\infty}}\right] d \tau\right)
\end{aligned}
$$

with

$$
V(t)=\int_{0}^{t}\left\|\partial_{x} v(\tau)\right\|_{B_{p, r}^{1 / p} \cap L^{\infty}}
$$

(3) If $f=v$, then for all $s>0$, (1) holds true when $V(t)=\int_{0}^{t}\left\|v_{x}(\tau)\right\|_{L^{\infty}} d \tau$.

(4) If $r<\infty$, then $f \in C\left([0, T] ; B_{p, r}^{s}\right)$. If $r=\infty$, then $f \in C\left([0, T] ; B_{p, 1}^{s^{\prime}}\right)$ for all $s^{\prime}<s$.

Lemma 2.4 (Existence and uniqueness) Let $p, r, s, f_{0}$ and $F$ be as in the statement of Lemma 2.3. Assume that $v \in L^{\rho}\left(0, T ; B_{\infty, \infty}^{-M}\right)$ for some $\rho>1$ and $M>0$ and $v_{x} \in L^{1}\left(0, T ; B_{p, r}^{s-1}\right)$ if $s>1+\frac{1}{p}$ or $s=1+\frac{1}{p}$ and $r=1$ and $v_{x} \in L^{1}\left(0, T ; B_{p, \infty}^{1 / p} \cap L^{\infty}\right)$ if $s<1+\frac{1}{p}$. Then problem (2.2) and (2.3) has a unique solution $f \in L^{\infty}\left(0, T ; B_{p, r}^{s}\right) \cap\left(\bigcap_{s^{\prime}<s} C\left([0, T] ; B_{p, 1}^{s^{\prime}}\right)\right)$ and the inequalities of Lemma 2.3 can hold true. Moreover, if $r<\infty$, then $f \in C\left([0, T] ; B_{p, r}^{s}\right)$.

\section{Proof of Theorem 1.1}

By using the following six steps, we will complete the proof of Theorem 1.1.

First step: Approximate solution. We will construct a solution with the aid of a standard iterative process. Starting from $u^{(0)}:=0$, by the inductive method and solving the following linear transport equation (3.1) and (3.2), we derive a sequence of smooth functions $\left(u^{(n)}\right)_{n \in \mathbf{N}}$

$$
\begin{aligned}
{\left[\partial_{t}+\left(u^{(n)}\right)^{2} \partial_{x}\right] u^{(n+1)}=} & -P_{1}(D)\left[\frac{4}{5}\left(u^{(n)}\right)^{5}-\frac{1}{3}\left(u^{(n)}\right)^{3}+\frac{3}{2} u^{(n)}\left(u_{x}^{(n)}\right)^{2}\right] \\
& -P_{2}(D)\left[\frac{1}{2}\left(u_{x}^{(n)}\right)^{3}\right], \\
u^{(n+1)}(x, 0)=u_{0}^{(n+1)}= & S_{n+1} u_{0} .
\end{aligned}
$$

It is easily checked that $S_{n+1} u_{0} \in B_{p, r}^{\infty}$, by using Lemma 2.4 and the inductive method, for all $n \in N$, we have that (3.1) and (3.2) has a global solution which belongs to $C\left(\mathbf{R}^{+}, B_{p, r}^{\infty}\right)$.

Second step: Uniform bounds. We will prove

$$
\begin{aligned}
\left\|u^{(n+1)}(t)\right\|_{B_{p, r}^{s}} \leq & e^{C \int_{0}^{t}\left\|u^{(n)}\right\|_{B_{p}^{s}, r}^{2} d \tau}\left\|u_{0}\right\|_{B_{p, r}^{s}}+\frac{C}{2} \int_{0}^{t} e^{C \int_{\tau}^{t}\left\|u^{(n)}\right\|_{B_{p}^{s}, r}^{2} d \tau}\left\|u^{(n)}\right\|_{B_{p, r}^{s}}^{3} d \tau \\
& +\frac{C}{2} \int_{0}^{t} e^{C \int_{\tau}^{t}\left\|u^{(n)}\right\|_{B_{p}^{s} r}^{2} d \tau}\left\|u^{(n)}\right\|_{B_{p, r}^{s}}^{5} d \tau
\end{aligned}
$$

for all $n \in \mathbf{N}$. 
Combining (2.4) of Lemma 2.3 with (3.1), we have

$$
\begin{aligned}
\left\|u^{(n+1)}(t)\right\|_{B_{p, r}^{s}} \leq & e^{C \int_{0}^{t}\left\|\left(\left(u^{(n)}\right)^{2}\right)\left(t^{\prime}\right)\right\|_{B_{p, r}^{s}} d t^{\prime}}\left\|u_{0}\right\|_{B_{p, r}^{s}} \\
& +\int_{0}^{t} e^{C \int_{\tau}^{t}\left\|\left(\left(u^{(n)}\right)^{2}\right)\left(t^{\prime}\right)\right\|_{B_{p, r}^{s}} d t^{\prime}}\left\|F\left(u^{(n)}, u_{x}^{(n)}\right)\right\|_{B_{p, r}^{s}} d \tau
\end{aligned}
$$

where

$$
F\left(u^{n}, u_{x}^{n}\right)=P_{1}(D)\left[\frac{4}{5}\left(u^{(n)}\right)^{5}-\frac{1}{3}\left(u^{(n)}\right)^{3}+\frac{3}{2} u^{(n)}\left(u_{x}^{(n)}\right)^{2}\right]+P_{2}(D)\left[\frac{1}{2}\left(u_{x}^{(n)}\right)^{3}\right] .
$$

When $s>\max \left\{1+\frac{1}{p}, \frac{3}{2}\right\}$, by using (4) in Lemma 2.2, we have

$$
\begin{aligned}
& \left\|\left(\left(u^{(n)}\right)^{2}\right)\left(t^{\prime}\right)\right\|_{B_{p, r}^{s}} \leq C\left\|\left(u^{(n)}\right)\left(t^{\prime}\right)\right\|_{B_{p, r}^{s}}^{2} \\
& \left\|F\left(u^{(n)}, u_{x}^{(n)}\right)\right\|_{B_{p, r}^{s}} \leq C\left[\left\|u^{(n)}\right\|_{B_{p, r}^{s}}^{3}+\left\|u^{(n)}\right\|_{B_{p, r}^{s}}^{5}\right] .
\end{aligned}
$$

Combining (3.6)-(3.7) with (3.4), we have (3.3).

Let $T>0$ satisfy

$$
\begin{aligned}
& T<\min \left\{\frac{1}{16 C\left\|u_{0}\right\|_{B_{p, r}^{s}}^{2}}, \frac{1}{C}\right\}, \\
& \left\|u^{(n)}(t)\right\|_{B_{p, r}^{s}} \leq \frac{2\left\|u_{0}\right\|_{B_{p, r}^{s}}}{\left(1-16 C\left\|u_{0}\right\|_{B_{p, r}^{s}}^{2} t\right)^{1 / 2}} .
\end{aligned}
$$

By using (3.9), we have

$$
e^{C \int_{\tau}^{t}\left\|u^{(n)}\right\|_{B_{p, r}^{s}}^{2}\left(t^{\prime}\right) d t^{\prime}} \leq e^{-\frac{1}{4} \int_{\tau}^{t} \frac{d\left(1-16 C\left\|u_{0}\right\|_{B_{p, r}^{s}}^{2} t^{\prime}\right)}{1-16 C\left\|u_{0}\right\|_{B_{p, r}^{s}}^{2} t^{\prime}}}=\left(\frac{1-16 C\left\|u_{0}\right\|_{B_{p, r}^{s}}^{2} \tau}{1-16 C\left\|u_{0}\right\|_{B_{p, r}^{s} t}^{2} t}\right)^{\frac{1}{4}} .
$$

When $\tau=0$ in (3.10), we have

$$
e^{C U^{n}(t)} \leq\left(\frac{1}{1-16 C\left\|u_{0}\right\|_{B_{p, r}^{s}}^{2} t}\right)^{1 / 4}
$$

By using (3.10) and (3.9), we have

$$
\begin{aligned}
& \frac{C}{2} \int_{0}^{t} e^{C \int_{\tau}^{t}\left\|u^{(n)}\left(t^{\prime}\right)\right\|_{B_{p, r}^{s}}^{2} d t^{\prime}}\left\|u^{(n)}(\tau)\right\|_{B_{p, r}^{s}}^{5} d \tau \\
& \quad \leq\left(1-16 C\left\|u_{0}\right\|_{B_{p, r}^{s}}^{2} t\right)^{-\frac{1}{4}} \int_{0}^{t} \frac{16 C\left\|u_{0}\right\|_{B_{p, r}^{s}}^{5}}{\left(1-16 C\left\|u_{0}\right\|_{B_{p, r}^{s}}^{2} \tau\right)^{\frac{9}{4}}} d \tau \\
& \quad=\frac{4}{5}\left\|u_{0}\right\|_{B_{p, r}^{s}}^{3}\left(1-16 C\left\|u_{0}\right\|_{B_{p, r}^{s}}^{2} t\right)^{-\frac{1}{4}}\left[\left(1-16 C\left\|u_{0}\right\|_{B_{p, r}^{s}}^{2} t\right)^{-\frac{5}{4}}-1\right] .
\end{aligned}
$$

With the aid of the mean value theorem, we have

$$
\left[\left(1-16 C\left\|u_{0}\right\|_{B_{p, r}^{s}}^{2} t\right)^{-\frac{5}{4}}-1\right]=20 C\left\|u_{0}\right\|_{B_{p, r}^{s}}^{2} t(1-\xi)^{-9 / 4},
$$


where

$$
16 C\left\|u_{0}\right\|_{B_{p, r}^{s}}^{2} t<\xi<1
$$

Combining (3.13) with (3.12), we have that

$$
\frac{C}{2} \int_{0}^{t} e^{C \int_{\tau}^{t}\left\|u^{(n)}\left(t^{\prime}\right)\right\|_{B_{p}^{s}, r}^{2}} d t^{\prime}\left\|u^{(n)}(\tau)\right\|_{B_{p, r}^{s}}^{5} d \tau \leq \frac{16\left\|u_{0}\right\|_{B_{p, r}^{s}}^{5} C t}{\left(1-16 C\left\|u_{0}\right\|_{B_{p, r}^{s}}^{2} t\right)^{5 / 2}} .
$$

Inserting (3.10)-(3.14) into (3.3) leads to

$$
\left\|u^{(n+1)}(t)\right\|_{B_{p, r}^{s}} \leq \frac{2\left\|u_{0}\right\|_{B_{p, r}^{s}}}{\left(1-16 C\left\|u_{0}\right\|_{B_{p, r}^{s}}^{2} t\right)^{1 / 2}}
$$

Consequently, $\left(u^{(n)}\right)_{n \in N}$ is uniformly bounded in $C\left([0, T] ; B_{p, r}^{s}\right)$. By using the fact that $B_{p, r}^{s-1}$ with $s>\max \left\{1+\frac{1}{p}, \frac{3}{2}\right\}$ is an algebra and $B_{p, r}^{s} \hookrightarrow B_{p, r}^{s-1}$ as well as the definition of the Besov spaces $B_{p, r}^{s}$, we derive that

$$
\begin{aligned}
\left\|\left(u^{(n)}\right)^{2} u_{x}^{(n+1)}\right\|_{B_{p, r}^{s-1}} & \leq C\left\|\left(u^{(n)}\right)^{2}\right\|_{B_{p, r}^{s-1}}\left\|u_{x}^{(n+1)}\right\|_{B_{p, r}^{s-1}} \\
& \leq C\left\|u^{(n)}\right\|_{B_{p, r}^{s}}^{2}\left\|u^{(n+1)}\right\|_{B_{p, r}^{s}} \\
& \leq \frac{8 C\left\|u_{0}\right\|_{B_{p, r}^{s}}^{3}}{\left(1-16 C\left\|u_{0}\right\|_{B_{p, r}^{s}}^{2} t\right)^{\frac{3}{2}}} .
\end{aligned}
$$

Since $s>\max \left\{1+\frac{1}{p}, \frac{3}{2}\right\}$, which leads to that $B_{p, r}^{s-1}$ is an algebra, by using the $S^{-1}$-multiplier property of $P_{1}(D)$ and the $S^{-2}$-multiplier property of $P_{2}(D)$ as well as (3.8), we have

$$
\begin{aligned}
& \left\|F\left(u^{(n)}, u_{x}^{(n)}\right)\right\|_{B_{p, r}^{s-1}} \leq \frac{C}{2}\left[\left\|u^{(n)}\right\|_{B_{p, r}^{s}}^{3}+\left\|u^{(n)}\right\|_{B_{p, r}^{s}}^{5}\right] \\
& \quad \leq 4 C\left[\frac{\left\|u_{0}\right\|_{B_{p, r}^{s}}^{3}}{\left(1-16 C\left\|u_{0}\right\|_{B_{, r}^{s}}^{2} t\right)^{\frac{3}{2}}}+\frac{4\left\|u_{0}\right\|_{B_{p, r}^{s}}^{5}}{\left(1-16 C\left\|u_{0}\right\|_{B_{p}^{s}, r}^{2} t\right)^{\frac{5}{2}}}\right] .
\end{aligned}
$$

Consequently, combining (3.1) with (3.16) and (3.17), we derive that

$$
\left\|u_{t}^{(n+1)}\right\|_{B_{p, r}^{s-1}} \leq 4 C\left[\frac{3\left\|u_{0}\right\|_{B_{p, r}^{s}}^{3}}{\left(1-16 C\left\|u_{0}\right\|_{B_{p, r}^{s}}^{2} t\right)^{\frac{3}{2}}}+\frac{4\left\|u_{0}\right\|_{B_{p, r}^{s}}^{5}}{\left(1-16 C\left\|u_{0}\right\|_{B_{p, r}^{s}}^{2} t\right)^{\frac{5}{2}}}\right]
$$

which yields $\left(u^{(n)}\right)_{n} \in C\left([0, T] ; B_{p, r}^{s}\right) \cap C^{1}\left([0, T] ; B_{p, r}^{s-1}\right)$.

Third step: Convergence. We will derive that $\left(u^{(n)}\right)_{n}$ is a Cauchy sequence in $C\left([0, T] ; B_{p, r}^{s-1}\right)$.

For $m, n \in N$, from (3.1), we have

$$
\left(u^{(n+m+1)}-u^{(n+1)}\right)_{t}+\left(u^{(n+m)}\right)^{2}\left(u^{(n+m+1)}-u^{(n+1)}\right)_{x}=\sum_{k=1}^{5} T_{k},
$$


where

$$
\begin{aligned}
& T_{1}=-\frac{4}{5} P_{1}(D)\left[\left(u^{(n+m)}\right)^{5}-\left(u^{(n)}\right)^{5}\right], \\
& T_{2}=\frac{1}{3} P_{1}(D)\left[\left(u^{(n+m)}\right)^{3}-\left(u^{(n)}\right)^{3}\right], \\
& T_{3}=-\frac{3}{2} P_{1}(D)\left[\left(u^{n+m}-u^{n}\right)\left(u_{x}^{(n+m)}\right)^{2}\right], \\
& T_{4}=-\frac{3}{2} P_{1}(D)\left[\left(u_{x}^{(n+m)}\right)^{2}-\left(u_{x}^{(n)}\right)^{2}\right], \\
& T_{5}=-\frac{1}{2} P_{2}(D)\left[\left(u^{(n+m)}\right)_{x}^{3}-\left(u^{(n)}\right)_{x}^{3}\right], \\
& T_{6}=\left[\left(u^{(n+m)}\right)^{2}-\left(u^{(n)}\right)^{2}\right] u_{x}^{(n+1)} .
\end{aligned}
$$

When $s>\max \left\{1+\frac{1}{p}, \frac{3}{2}\right\}$, by using the $S^{-1}$ multiplier property of $P_{1}(D)$, the $S^{-2}$ multiplier property of $P_{2}(D)$ and $B_{p, r}^{s-1} \hookrightarrow B_{p, r}^{s-2}$, we have

$$
\left\|T_{j}\right\|_{B_{p, r}^{s-1}} \leq C\left\|u^{(n+m)}-u^{(n)}\right\|_{B_{p, r}^{s-1}}
$$

where $1 \leq j \leq 6, j \in \mathbf{N}$. Since $\forall n \in N$, we have

$$
\left\|u^{(n)}\right\|_{B_{p, r}^{s}} \leq \frac{2\left\|u_{0}\right\|_{B_{p, r}^{s}}}{\left(1-16 C\left\|u_{0}\right\|_{B_{p, r}^{s}}^{2} t\right)^{\frac{1}{2}}} .
$$

By using (3.20), we have

$$
\begin{aligned}
\left\|\sum_{j=1}^{6} T_{j}\right\|_{B_{p, r}^{s, 1}} & \leq \sum_{j=1}^{6}\left\|T_{j}\right\|_{B_{p, r}^{s, 1}} \\
& \leq C\left\|u^{(n+m)}-u^{(n)}\right\|_{B_{p, r}^{s-1}} \frac{4\left\|u_{0}\right\|_{B_{p, r}^{s}}^{2}}{1-16 C\left\|u_{0}\right\|_{P_{p, r}^{s}}^{2} t} .
\end{aligned}
$$

When $s \neq 2+\frac{1}{p}$, from Lemma 2.4 and (3.20), we have

$$
\begin{aligned}
& \|\left(u^{(n+m+1)}-u^{(n+1)}(t) \|_{B_{p, r}^{s-1}}\right. \\
& \leq e^{C W^{(n+m)}(t)}\left\|\left(u^{(n+m+1)}-u^{(n+1)}\right)(\cdot, 0)\right\|_{B_{p, r}^{s-1}} \\
& \quad+C \int_{0}^{t} e^{C W^{(n+m)}(t)-C W^{(n+m)}(\tau)} \sum_{j=1}^{6}\left\|T_{j}\right\|_{B_{p, r}^{s-1}} d \tau,
\end{aligned}
$$

where

$$
W^{n+m}(t)=\int_{0}^{t}\left\|\partial_{x}\left(u^{(n+m)}\right)^{2}(\tau)\right\|_{B_{p, r}^{\frac{1}{p}} \cap L^{\infty}} d \tau
$$


if $s-1<1+\frac{1}{p}$ and

$$
W^{n+m}(t)=\int_{0}^{t}\left\|\partial_{x}\left(u^{(n+m)}\right)^{2}(\tau)\right\|_{B_{p, r}^{s-2}} d \tau
$$

if $s-1>1+\frac{1}{p}$. From (3.23), if $s-1<1+\frac{1}{p}$, by using $B_{p, r}^{s-1} \hookrightarrow L^{\infty}$ with $s>1+\frac{1}{p}$, we have

$$
W^{n+m}(t) \leq C \int_{0}^{t}\left\|u^{(n+m)}(\tau)\right\|_{B_{p, r}^{s}}^{2} d \tau
$$

From (3.25), if $s-1>1+\frac{1}{p}$, we have

$$
W^{n+m}(t) \leq C \int_{0}^{t}\left\|u^{(n+m)}(\tau)\right\|_{B_{p, r}^{s}}^{2} d \tau
$$

It is easily showed that

$$
\left\|\sum_{q=n+1}^{n+m} \Delta_{q} u_{0}\right\|_{B_{p, r}^{s-1}} \leq C 2^{-n}\left\|u_{0}\right\|_{B_{p, r}^{s-1}}
$$

Inserting (3.25)-(3.27) into (3.22), we have

$$
\begin{aligned}
& \left\|\left(u^{(n+m+1)}-u^{(n+1)}\right)(t)\right\|_{B_{p, r}^{s-1}} \\
& \quad \leq C_{T}\left(2^{-n}+\int_{0}^{t}\left\|\left(u^{(n+m)}-u^{(n)}\right)(\tau)\right\|_{B_{p, r}^{s-1}} d \tau\right) .
\end{aligned}
$$

We define

$$
\begin{aligned}
& W_{n, k}(t)=\left\|\left(u^{(n+m)}-u^{(n)}\right)(t)\right\|_{B_{p, r}^{s-1}}, \\
& W_{n}(t)=\sup _{m \in \mathbf{N}} W_{n, m}(t), \\
& \widetilde{W}(t)=\limsup _{n \rightarrow \infty} W_{n}(t) .
\end{aligned}
$$

Combining (3.28) with (3.29)-(3.30), we have that

$$
W_{n+1}(t) \leq C \int_{0}^{t} W_{n}(\tau) d \tau
$$

From (3.31) and (3.32), by using the Fatou lemma, we have that

$$
\widetilde{W}(t) \leq C \int_{0}^{t} \widetilde{W}(\tau) d \tau
$$

Applying the Gronwall inequality to (3.33), we have that

$$
\widetilde{W}(t) \leq e^{C} \widetilde{W}(0)
$$


From (3.31), we have that $\widetilde{W}(0)=0$. Thus, $\widetilde{W}(t)=0$. Consequently, $\left(u^{(n)}\right)_{n}$ is a Cauchy sequence in $C\left([0, T] ; B_{p, r}^{s-1}\right)$; moreover, $\left(u^{(n)}\right)_{n}$ is convergent to some limit function $u \in$ $C\left([0, T] ; B_{p, r}^{s-1}\right)$.

When $s=2+\frac{1}{p}$, by using (6) of Lemma 2.2, we derive that

$$
\begin{aligned}
& \left\|\left(u^{(n+m+1)}-u^{(n+1)}\right)(t)\right\|_{L_{T}^{\infty} B_{p, r}^{s-1}} \\
& \quad=\left\|\left(u^{(n+m+1)}-u^{(n+1)}\right)(t)\right\|_{L_{T}^{\infty} B_{p, r}^{1+\frac{1}{p}}} \\
& \leq\left\|\left(u^{(n+m+1)}-u^{(n+1)}\right)(t)\right\|_{L_{T}^{\infty} B_{p, r}^{s_{1}}}^{\theta}\left\|\left(u^{(n+m+1)}-u^{(n+1)}\right)(t)\right\|_{L_{T}^{\infty} B_{p, r}^{s_{2}}}^{1-\theta} \\
& \leq\left\|\left(u^{(n+m+1)}-u^{(n+1)}\right)(t)\right\|_{B_{p, r}^{1+\frac{1}{p}}}^{\theta}\left[\left\|u^{(n+m+1)}\right\|_{B_{p, r}^{2+\frac{1}{p}}}+\left\|u^{(n+1)}(t)\right\|_{B_{p, r}^{2+\frac{1}{p}}}\right]^{1-\theta} \\
& \leq\left(C_{T}^{\prime}\right)^{\theta} 2^{-\theta n}\left[\left\|u^{(n+m+1)}\right\|_{B_{p, r}^{2+\frac{1}{p}}}+\left\|u^{(n+1)}(t)\right\|_{B_{p, r}^{2+\frac{1}{p}}}\right]^{1-\theta},
\end{aligned}
$$

where

$$
s_{1} \in\left(\max \left(1+\frac{1}{p}, \frac{3}{2}\right)-1,1+\frac{1}{p}\right), \quad s_{2} \in\left(1+\frac{1}{p}, 2+\frac{1}{p}\right) .
$$

Consequently, $\left(u^{(n)}\right)_{n}$ is a Cauchy sequence in $C\left([0, T] ; B_{p, r}^{s-1}\right)$ and $\left(u^{(n)}\right)_{n}$ converges to some limit function $u \in C\left([0, T] ; B_{p, r}^{s-1}\right)$.

Fourth step: Existence of solution in $E_{p, r}^{s}(T)$. Existence of solution $E_{p, r}^{s}(T)$ can be proved similarly to [11].

Fifth step: Uniqueness of solution. We consider case $s \neq 2+\frac{1}{p}$ and case $s=2+\frac{1}{p}$, respectively. In fact, this can be proved similarly to [14].

Sixth step: Continuity with respect to the initial data. Continuity with respect to the initial data can be proved similarly to [6].

\section{Proof of Theorem 1.2}

Since $B_{2,1}^{3 / 2}$ and $B_{2,1}^{1 / 2}$ are Banach algebras, by using a proof similar to (3.3), we can prove that

$$
\begin{aligned}
\left\|u^{(n+1)}(t)\right\|_{B_{2,1}^{3 / 2}} \leq & e^{C \int_{0}^{t}\left\|u^{(n)}\right\|_{B_{2,1}^{3 / 2}}^{2} d \tau}\left\|u_{0}\right\|_{B_{2,1}^{3 / 2}}+\frac{C}{2} \int_{0}^{t} e^{C \int_{\tau}^{t}\left\|u^{(n)}\right\|_{B_{2,1}^{3 / 1}}^{2} d \tau}\left\|u^{(n)}\right\|_{B_{2,1}^{3 / 2}}^{3} d \tau \\
& +\frac{C}{2} \int_{0}^{t} e^{C \int_{\tau}^{t}\left\|u^{(n)}\right\|_{B_{2,1}^{3 / 2}}^{2} d \tau}\left\|u^{(n)}\right\|_{B_{2,1}^{3 / 2}}^{5} d \tau
\end{aligned}
$$

for all $n \in \mathbf{N}$. We assume that

$$
\begin{aligned}
& T<\min \left\{\frac{1}{16 C\left\|u_{0}\right\|_{B_{2,1}^{3 / 2}}^{2}}, \frac{1}{C}\right\}, \\
& \left\|u^{(n)}(t)\right\|_{B_{2,1}^{3 / 2}} \leq \frac{2\left\|u_{0}\right\|_{B_{2,1}^{3 / 2}}}{\left(1-16 C\left\|u_{0}\right\|_{B_{2,1}^{3 / 2}}^{2} t\right)^{1 / 2}} .
\end{aligned}
$$

By a proof similar to (3.15), we can prove that

$$
\left\|u^{(n+1)}(t)\right\|_{B_{2,1}^{3 / 2}} \leq \frac{2\left\|u_{0}\right\|_{B_{2,1}^{3 / 2}}}{\left(1-16 C\left\|u_{0}\right\|_{B_{2,1}^{3 / 2}}^{2} t\right)^{1 / 2}} .
$$


Thus, $\left(u^{(n)}\right)_{n}$ is uniformly bounded in $B_{2,1}^{3 / 2}$. From (3.1), by using (4) in Lemma 2.2, we can prove that $\left(u_{t}^{(n)}\right)_{n}$ is uniformly bounded with respect to $n$ in $B_{2,1}^{1 / 2}$.

Consequently, $\left(u^{(n)}\right)_{n} \in C\left([0, T] ; B_{2,1}^{3 / 2}\right) \cap C^{1}\left([0, T] ; B_{2,1}^{1 / 2}\right)$.

We define

$$
\begin{aligned}
& \rho_{n, k}(t)=\left\|\left(u^{(n+m)}-u^{(n)}\right)(t)\right\|_{B_{2, \infty}^{1 / 2}}, \\
& \rho_{n}(t)=\sup _{m \in \mathbf{N}} \rho_{n, m}(t), \\
& \tilde{\rho}(t)=\limsup _{n \rightarrow \infty} \rho_{n}(t) .
\end{aligned}
$$

By a proof similar to [15], we derive that

$$
\widetilde{\rho}(t)=0
$$

and with the aid of $(2.1)$, we can prove that $\left(u^{(n)}\right)_{n}$ is a Cauchy sequence in $C\left([0, T] ; B_{2,1}^{1 / 2}\right)$.

The rest of Theorem 1.2 can be proved similarly to $[13,15]$.

\section{Competing interests}

We declare that we have no competing interests.

\section{Authors' contributions}

All authors read and approved the final manuscript.

\section{Author details}

${ }^{1}$ College of Mathematics and Information Science, Henan Normal University, Xinxiang, Henan 453007, P.R. China. ${ }^{2}$ Henan Vocational College of Agriculture, Zhengzhou, Henan 451450, P.R. China.

\section{Acknowledgements}

We would like to thank the reviewers for careful reading and valuable comments on the original draft.

Received: 18 March 2014 Accepted: 26 June 2014 Published online: 24 September 2014

\section{References}

1. Zhao, L, Zhou, SG: Symbolic analysis and exact travelling wave solution to a new modified Novikov equation. Appl. Math. Comput. 217, 590-598 (2010)

2. Novikov, VS: Generalizations of the Camassa-Holm equation. J. Phys. A 42, 342002 (2009)

3. Ni, L, Zhou, Y: Well-posedness and persistence properties for the Novikov equation. J. Differ. Equ. 250, $3002-3021$ (2011)

4. $\mathrm{Wu}, \mathrm{XL}$, Yin, ZY: Global weak solutions for the Novikov equation. J. Phys. A, Math. Theor. 44, 055202 (2011)

5. $\mathrm{Wu}, \mathrm{XL}$, Yin, ZY: A note on the Cauchy problem for the Novikov equation. Appl. Anal. 92, 1116-1137 (2013)

6. Yan, W, Li, YS, Zhang, YM: The Cauchy problem for the integrable Novikov equation. J. Differ. Equ. 253, 298-318 (2012)

7. Yan, W, Li, YS, Zhang, YM: The Cauchy problem for the Novikov equation. Nonlinear Differ. Equ. Appl. 20, 1157-1169 (2013)

8. Lai, SY: Global weak solutions to the Novikov equation. J. Funct. Anal. 265, 520-544 (2013)

9. Li, Y, Yan, W: Well-posedness and ill-posedness of KdV equation with higher dispersion. J. Math. Anal. Appl. 416, 647-658 (2014)

10. Danchin, R: A few remarks on the Camassa-Holm equation. Differ. Integral Equ. 14, 953-988 (2001)

11. Danchin, R: Fourier Analysis Method for PDEs. Lecture Notes, vol. 14 (2005)

12. Chemin, JY: Localization in Fourier space and Navier-Stokes. In: Phase Space Analysis of Partial Differential Equations. CRM Series, pp. 53-136. Scuola Norm. Sup., Pisa (2004)

13. Danchin, R: A note on well-posedness for Camassa-Holm equation. J. Differ. Equ. 192, 429-444 (2003)

14. Yan, W, Li, YS, Zhang, YM: The Cauchy problem for the generalized Camassa-Holm equation. Appl. Anal. 93, 1358-1381 (2014)

15. Yan, W, Li, YS, Zhang, YM: The Cauchy problem for the generalized Camassa-Holm equation in Besov space. J. Differ. Equ. 256, 2876-2901 (2014) 